\title{
Virtual Biomaterials Lab During COVID-19 Pandemic
}

\author{
Jonathan A. Gerstenhaber (i) and Yah-el Har-el (1) \\ Department of Bioengineering, Temple University, Philadelphia, PA, USA \\ (Received 30 June 2020; accepted 11 December 2020; published online 18 February 2021).
}

\section{CHALLENGE STATEMENT}

This past Spring 2020 our department offered a required lab, Biomaterials Lab. The class consists of 1 $\mathrm{h}$ of lecture for all students on Monday and $2 \mathrm{~h}$ of lab on both Tuesdays and Thursdays ( 3 sections, 86 students). At the time the university closed for COVID19, we had planned cell culture experiments demonstrating the effects of mechanical properties and diffusivity. Our challenge was twofold. We needed to create new laboratory materials without including any cell work while demonstrating the structure-property relationships as applied to properties of biomaterials and relevance to biology and biomedicine. We also needed to deliver these new labs in a virtual setting, which was new to both the students and instructors. Virtual materials labs have been reported, as there has been interest in developing lab courses where students can learn and make mistakes before using equipment or as an enhancement to a hands on lab. ${ }^{4,9}$ Application of these past models was difficult as we could not combine hands-on experience, and many required the students have programming expertise beyond that which the students in our program have at this time in the curriculum. ${ }^{3}$

\section{NOVEL INITIATIVE}

We chose to complete the remaining labs in SolidWorks including the Simulation functionality and in MATLAB including the PDE toolbox (3 full labs). The students were given asynchronous videos explaining the lab and the software as well as written lab

Address correspondence to Yah-el Har-el, Department of Bioengineering, Temple University, Philadelphia, PA, USA. Electronic mail: yahel@temple.edu instructions. The materials for these labs are available as Supplemental Material.

\section{Simulations}

Our first lab online was a continuation of an in-class activity centered around design of bone cements. It was intended to include mechanical testing and analysis of data from an MTS instrument. TAs completed material testing and documented it with photos and short video demos to simulate the in-class experience. The raw data collected (compressing bone cement samples) was sent to the class for analysis in Microsoft Excel.

The second lab required rendering standard test specimen (dog bones, rectangular prisms) in SolidWorks, and compression, tension, and 3-point bending tests similar to those originally meant to be completed in person on biological specimen. Students analyzed the effects of materials in the models by applying properties they researched on MatWeb, a materials database. ${ }^{6}$ While students could not prepare and test biological specimen or view complex modulus, this allowed students to compare more types of mechanical tests and data from different sources or created using different methods. It's been reported that virtual labs can enhance students' understanding of the material, ${ }^{4,9}$ in this case, it would broaden their appreciation of the diversity of mechanical properties of bone, tendon, and cartilage.

The next lab was also in SolidWorks and focused on the rendering of a human femoral hip replacement as a demonstration of the importance of material properties to biomechanics. A CT scan of a femur from the NIH 3D Print Exchange was prepared for use in SolidWorks (see Supplemental Material). To reduce computational requirements, preparation of the file included: import, resection of the lower half of the bone, and mesh simplification. The femoral head was 
also replaced with a sphere to serve as the starting location for implant design, and a marrow cavity was added. The model included simplifications to facilitate surgery and modeling, such as a constant cortical thickness. The students constructed simple implants using cross sections connected with the loft tool and reported on how material choice was important to safety, stress-shielding, and implant durability as visualized by stress, strain, and factor of safety.

Our final lab demonstrated the effects of hydrogel design on diffusivity and drug delivery using MATLAB as a replacement for a wet lab demonstrating the same. For 2D simulations of a single material, the PDE toolbox offers a guided GUI interface called the PDE Modeler App (command: pdetool). The interface includes a diffusion setting, helping to guide users with the definitions of terms, and there are detailed examples provided by Mathworks demonstrating use of the tool. While students were able to test the effect of diffusivity constants effectively, the more difficult aspect here is the successful calculation of such constants given a description of an experiment, a focus which is somewhat less relevant in a wet lab context where the focus was on biological responses to drug delivery.

For simulations of multiple materials (rate release membranes, for example) and graphing delivery vs time, the PDE toolbox programmatic methods were used. There is no guided 'diffusion' set of commands available in the PDE toolbox programmatic methods. A simplified mechanism exists for heat flow (which was leveraged due to similarity of the underlying equations) but results in some non-intuitive use of commands. As a result Matlab code prepared for this laboratory was provided which could functionally test a specific material and geometry (see Supplementary Material). Students were expected to paste the relevant code into MatLab and modify the parts corresponding to material and geometry as required. Through the use of the 'pdetool' drawing application, students were able to furnish personal designs including separate release coatings. For simplicity, the model provided was limited to drug concentrations below solubility.

\section{Delivery Tools}

Our students have had minimal exposure with SolidWorks and Matlab at this time, so we replaced the general Biomaterials lectures with lectures focused more specifically on the labs and navigating these two software programs. With Echo360 we were able to record PowerPoint material with the professor's notations simultaneous with the professor via webcam in order to increase a sense of 'face time'. When appropriate, either one of these video feeds were exchanged for the simulation tool being used, a function especially helpful when demonstrating new software. Students can pause and replay wherever they feel a need and vary play speed. This software also allows the instructors to see who had watched the video and to what degree they interacted with the material (such as viewing the slide deck), so trouble areas can be quickly identified.

The labs were designed to be done independently and asynchronously. In addition to the Echo360 video, they were also given step-by-step instructions. To help them with questions or concerns, each of the two instructors was available on Zoom for 3 office hours and 1 hour during the normal lab section per week. Students were instructed to attend any of the 'lab hours', not only those aligning with their original class time.

Solidworks and Matlab and required add-ons are available to our students for free. However, SolidWorks is available only on PC. Mac users were provided a custom VirtualBox Virtual Machine and detailed install instructions including screenshots and install size details to run Solidworks. To alleviate computing issues, students were also provided with a remote desktop system, which allowed them to run simulations on a university computer.

\section{Assessment Methods}

All labs included both individual and group assessments. Individual assessments took the form of Canvas quizzes with questions related to lab procedures and data interpretation. There was also a cumulative final built in a similar fashion. Group submissions included lab reports which required background research for the introduction and discussion. A summary of the grades achieved for the methods, results and discussion sections of the lab reports can be seen in Fig. 1, introduction, conclusion, and references did not demonstrate as strong a diversity across the course. The lab manuals also included goals and questions to be addressed in their report. All figures in this paper were prepared in JMP 14 (SAS Institute, NC, USA).

As a required course, we assess here "an ability to function effectively on a team... (ABET EAC 20192020 SO 5)" directly by the instructors and TAs. We wanted to continue group work since engineering knowledge can only be learned through the senses and social interactions. ${ }^{1}$ Peer assessments, added after we went virtual, were limited to a short survey of whether everyone contributed, whether there was someone who did very little work and whether there was someone who should not get credit because they did no work. We followed up with every team that answered the last two questions in the affirmative. This was very differ- 
ent compared to the original in-person method of grading live group dynamics with input from the instructors and TAs.

Student feedback on course performance was collected through four surveys. A short quiz asking about resources used and the final which included two open ended questions on how the student would have pre- ferred the class to run both online or otherwise, and multiple-choice Likert scale and open response surveys to provide end-of-semester feedback sent by the College and by the University. Student response was near $100 \%$ in our quiz and exam questions but the college and university administered surveys had lower responses (Table 1).

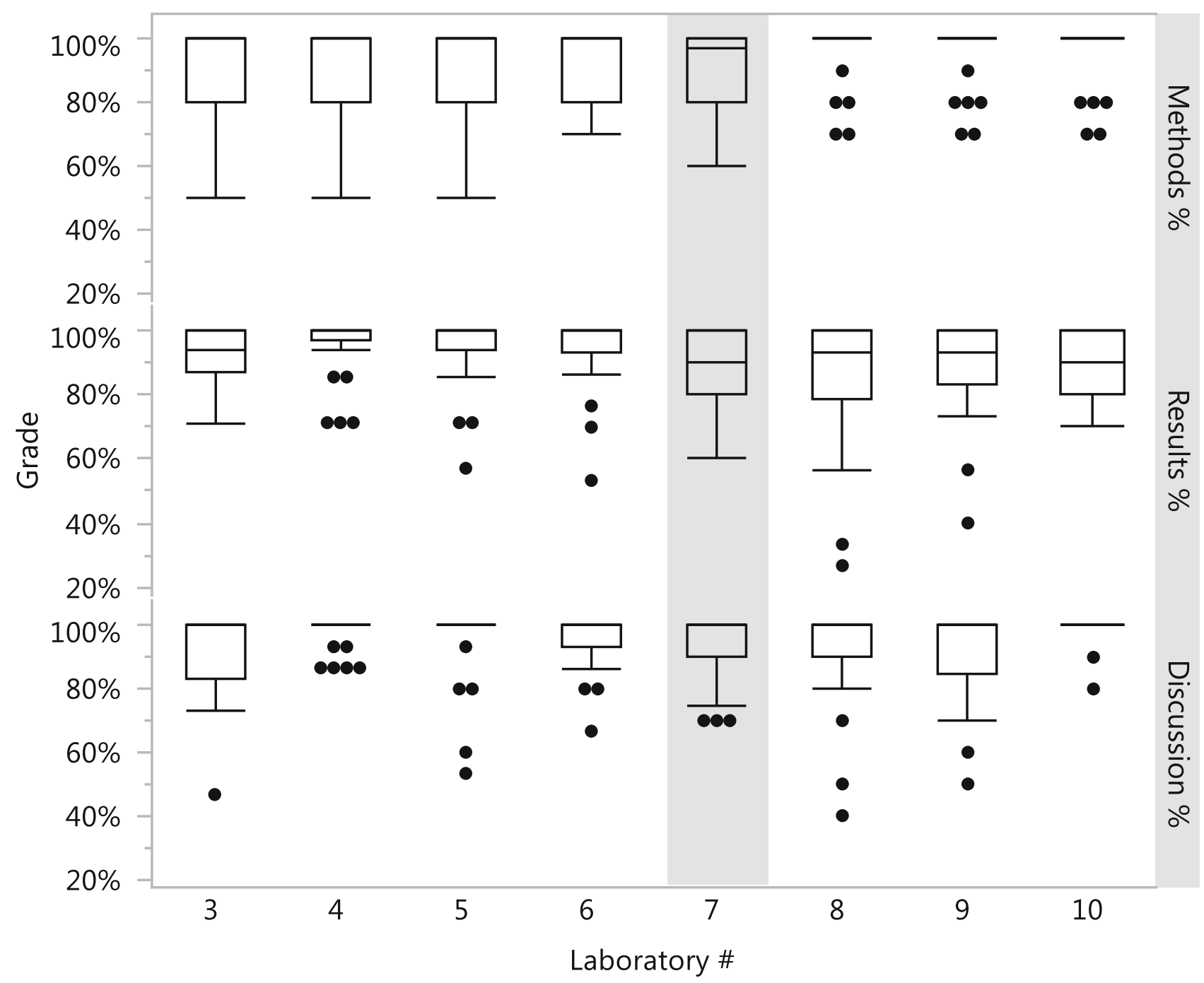

FIGURE 1. Box and whiskers plot of the grades received in the Discussion, Methods and Results sections of lab reports \#3-10. Labs \#1 and 2 were not included as students were still adjusting to the grading rubric. Halfway through lab 7 (shaded), we transitioned online. Lab report sections for the introduction, conclusion, and references did not demonstrate meaningful diversity across the course and are not included.

TABLE 1. Indirect assessment methods of this biomaterials lab course.

\begin{tabular}{lcc}
\hline & Response rate (\%) & Question format \\
\hline Quiz questions & 86 & Yes/no \\
Exam questions & 100 & Open response \\
College end of semester survey & 65 & Likert scale/open response \\
University end of semester survey & 74 & Likert scale/open response \\
\hline
\end{tabular}

Enrollment was 86 students in three sections. 


\section{REFLECTION}

The course objectives included basic cell culture techniques and we were able to offer 4 cell-based labs before going virtual. The prepared simulation laboratories were successful at meeting the remaining course learning objectives (see Table 2) which reflect many of the core competencies for BIOE labs. ${ }^{7}$ In a fully-remote semester, simulations of sterile technique, instrument use, and effects of materials on living tissue, would have to be better represented.

Individual quiz assessments did not differ significantly over the course, with the exception of the second Solidworks lab, which students found the most difficult. When looking at the grades students received on their lab reports during this time, we find that students showed poorer scores in their data analysis/results with the move to remote learning (Fig. 1). Through the questions asked in the final quiz and exam, we learned that only 17 of the 74 responders utilized the professors' or TAs' time online to ask for help or to ask questions about the labs. We believe a solution to this would be to conduct virtual labs synchronously so that students ask questions in real time and better understand what they are doing and confirm they are doing the experiment correctly. At the same time, we included online asynchronous lectures which the students could access repeatedly for virtual labs (8-10 being entirely delivered online). We believe this is the reason the students improved their scores in the methods and discussion of their lab reports (Fig. 1). Other sections that were graded (introduction, conclusion and references) did not have significant changes across hands-on and virtual labs. Planning for this course in Spring 2021 we will be including an asynchronous lecture with lab instructions prior to the synchronous lab time for in person and virtual labs.

During the end of semester surveys, several students expressed what they would like to see improved in the course (Fig. 2). Some comments that were specific to the virtual labs included more synchronous time ( $15 \%$ respondents) and more communication about additional computational services $(\sim 10 \%$ respondents). A smaller group of students mentioned a preference for the hands-on labs from the start of the semester, but many of these expressed understanding of the circumstances. There is a strong possibility we will need to conduct online lab courses in the future and there were definitely some aspects that worked and others that didn't. Students with a personal focus on mechanics or prosthetics found the material especially valuable and it built on skills they have been learning (CAD programming), while those with a more cellular focus often found the simulation requirements tedious or unnecessarily complicated.

The recorded lectures in Echo360 had a mixed response. Many students thought they were clear and well presented, but there was a cohort of students that preferred a live lecture so that they can ask questions immediately. Nearly $20 \%$ of students expressed dis-

TABLE 2. Specific aims of the virtual labs mapped to the course learning objectives.

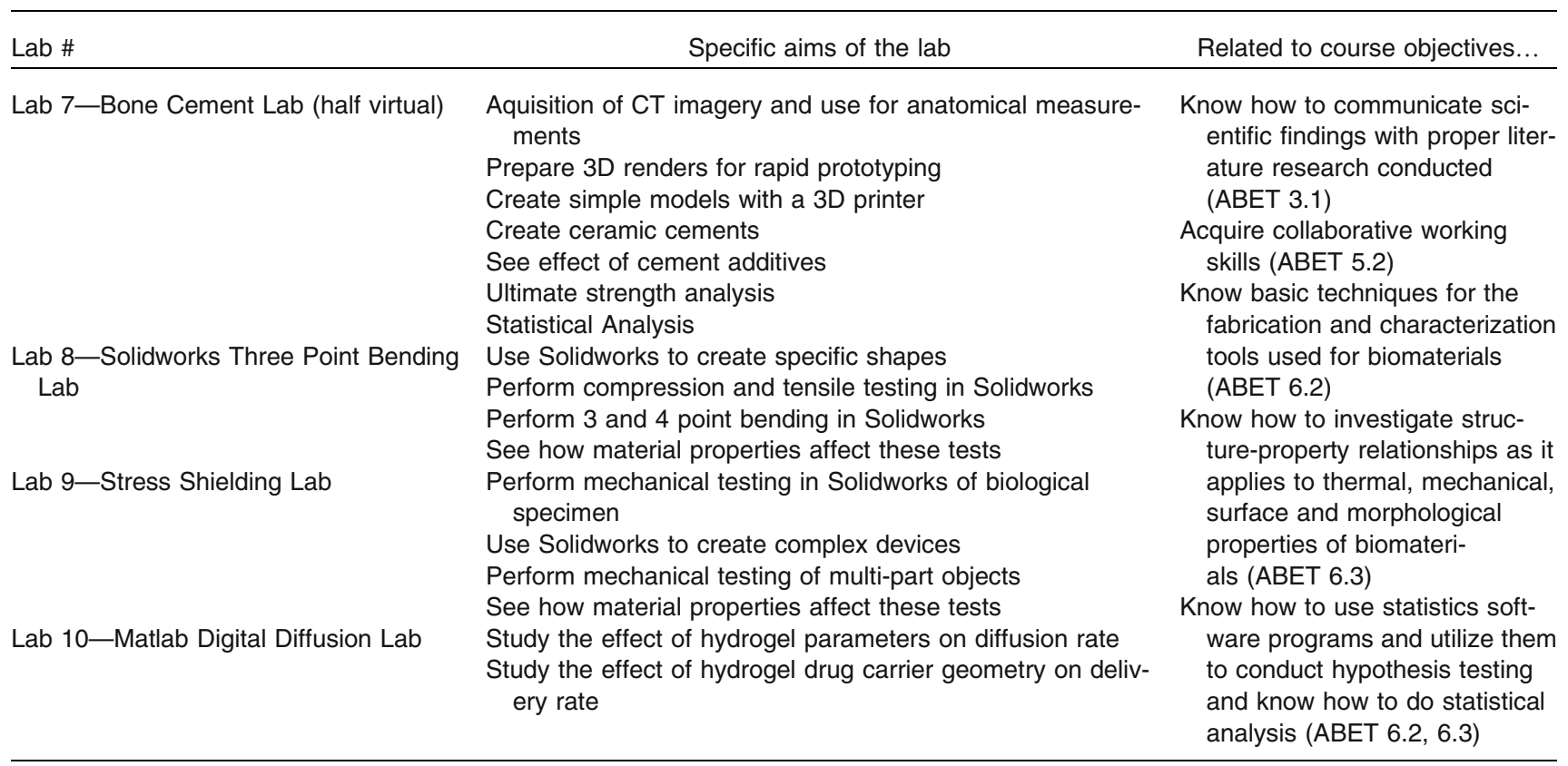




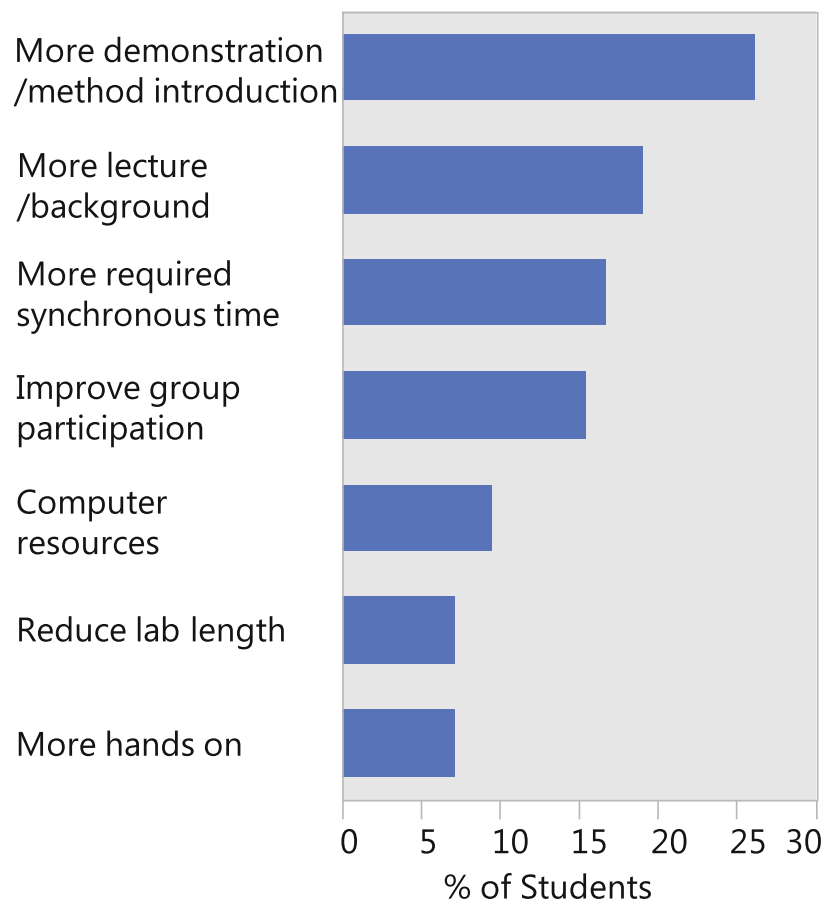

FIGURE 2. Student responses to the open ended question "How would you change the online aspect of the class if it needs to be offered online again?" put into general categories and what percentage of students felt that was an issue. The percentages are based on the students who responded to the open ended questions from the college wide end of semester survey. Some student responses indicated more than one category, and others included only positive comments, which is why the sum of the responses do not equal $100 \%$, nor is every student represented.

appointment that the biomaterials related lectures, which provided a more holistic background, were not continued when online due to the focus on use of the simulation tools. (Fig. 2) As such, we plan to continue such detailed backgrounds of the labs, provided asynchronously so that we can continue our weekly lecture with biomaterials background information. In the future instances of this course online, we would be able to plan out the lectures and lab instructions more evenly. We would also spread out the instruction of the software over more time as the most common request (over $25 \%$ of students) was for a more extensive demonstration and introduction to the use of the simulation methods. This may also improve the experience by students who have less programmatic background.

Students generally did not view the Echo360 instructions until shortly before the lab report was due, often not giving themselves enough time to complete the work or the ability to attend a Zoom session. This may have contributed to the difficulty many teams faced in encouraging participation from some members. The inclusion of required attendance during the virtual lab is planned for future online class with an initial instruction period followed by the student groups working in breakout rooms to address both of these issues.

While it is not practical to have every student submit an individual lab report, not doing so meant some students felt they could write an introduction and/or conclusion and have that be sufficient contribution to the lab report. Having self-selected teams did improve the ability of some teams to communicate but did not appear to keep students as responsible to the team as we expected. In the future, we would have each student individually collect data and submit it as a separate assignment. The group lab report would combine their data with the appropriate statistical analysis. The assessment protocol for virtual teamwork that we implemented was severely lacking. It was neither sufficiently rapid, nor sensitive, to allow us to react to team problems, let alone sufficiently assess performance on the team, though we were able to assess teamwork from the initial hands-on labs. In future, we could use peer evaluation assessment programs such as CATME $^{2,5}$ or $\operatorname{SPARK}_{\text {plus. }} 8,10$

A direct assessment of the students' ability to meet the course objectives in the form of the ABET outcomes indicated that all students met the course goals, as evaluated by their lab reports and the professors' and TAs' opinion of their team interactions. We have mapped the specific aims we set out to achieve in the virtual labs to the course objectives (Table 2). With the exception of hands on cell culture, all of our course learning objectives can be met by the new virtual labs that we introduced.

During the pandemic, we were able to offer simulations in areas of biomaterials relevant to the students, expanding their knowledge of Solidworks and Matlab, which will be beneficial in their Senior Design course. We have already seen the benefit of this in classes the students took in Fall 2020, where they were able to utilize skills in Solidworks in their Biodesign class. The majority of students found our approach beneficial and felt good about what they learned. Given a fully in person semester, we would continue including the content of these virtual labs in this course. The students' feedback led to the suggestions we made to change the delivery of these labs when the course goes online again in Spring 2021. Specifically, we will offer more required synchronous lab time for the students which we expect will give them more immediate feedback and foster more collaboration amongst the students. We will also maintain a biomaterial lecture in parallel with asynchronous lab demonstrations so that the videos presenting background are more distinguished by those presenting method. 


\section{SUPPLEMENTARY INFORMATION}

The online version of this article (https://doi.org/10. 1007/s43683-020-00045-6) contains supplementary material, which is available to authorized users.

\section{ACKNOWLEDGMENTS}

We would like to acknowledge the contributions of both Joseph Licata and Jennifer Patten in the running of this course and the generation of the data set used for Fig. 1.

\section{AUTHOR CONTRIBUTIONS}

$\mathrm{YH}$ and JAG were instructors in this course in Spring 2020 during the COVID-19 pandemic. YH wrote the majority of the article with contributions from JAG. JAG wrote the majority of the supplemental material with contributions from $\mathrm{YH}$.

\section{FUNDING}

Not applicable.

\section{DATA AVAILABILITY}

Supplemental course materials were designed by the authors.

\section{CODE AVAILABILITY}

Not applicable.

\section{CONFLICT OF INTEREST}

The authors declare that they have no conflict of interest.

\section{ETHICAL APPROVAL}

\section{CONSENT TO PARTICIPATE}

Not applicable.

\section{CONSENT FOR PUBLICATION}

Not applicable.

\section{REFERENCES}

${ }^{1}$ Brereton M. The role of hardware in learning engineering fundamentals: an empirical study of engineering design and product analysis activity $\mathrm{PhD}$ Stanford University, Stanford, CA, 1999.

${ }^{2}$ CATME Smarter Teamwork. http://info.catme.org. Accessed 17 Sept 2020

${ }^{3}$ Griffin L, Crockett R. AC 2007-2524: biomedical engineering simulation using visual basic macros in Microsoft Excel. In: Presented at the 2007 ASEE Annual Meeting.

${ }^{4}$ Hossain A, Fragomeni J. Integrating material science and processing into the undergraduate engineering \& science curriculum using the web. In: Presented at the 2004 ASEE Annual Conference, Salt Lake City, UT, 2004.

${ }^{5}$ Loughry M, Ohland M, Moore D. Development of a theory-based assessment of team member effectiveness. Educ Psychol Meas. 2007;67:505-24.

${ }^{6}$ Matweb Material Property Data. http://matweb.com. Accessed 17 Sept 2020.

${ }^{7}$ Perreault E, Litt M, Saterbak A. Educational methods and best practices in BME laboratories. Ann Biomed Eng. 2006;34:209-16.

${ }^{8}$ Spark Plus Flexible Self \& Peer Assessment and Feedback. https://sparkplus.com.au. Accessed 17 Sept 2020.

${ }^{9}$ Trumbower R, Enderle J. Virtual instruments in undergraduate biomedical engineering laboratories: a flexible, inexpensive, and responsive solution to preparing students for the "Real World". IEEE Eng Med Biol Mag. 2003;22:101-10.

${ }^{10}$ Willey K, Gardner K. Developing team skills with self- and peer assessment. Campus-Wide Inf Syst. 2009;26:365-78.

Publisher's Note Springer Nature remains neutral with regard to jurisdictional claims in published maps and institutional affiliations.

Not applicable. 